\title{
Centros de ciencia y museos científicos virtuales: teoría y práctica.
}

\author{
Marcelo Sabbatini \\ marcelo@sabbatini.com \\ Universidad de Salamanca
}

\section{Introducción}

El museo como la biblioteca cumple una misión de accesibilidad colectiva a productos de la creación humana, mediante la creación de un espacio de acumulación y concentración de acervo cultural de la comunidad. El acceso al museo equivale a la participación en una zona de construcción del conocimiento, a un proyecto social de diseminación de productos culturales. De ahí, que la visita a un museo deba formar parte del catálogo de acciones de formación. Precisamente la localización física del museo, junto a la real oferta de valores culturales, presenta los límites de acceso propios de la localización, junto a los que se corresponden con las limitaciones cognitivas de los visitantes. El valor cultural del museo, al confrontar su público real respecto a su público potencial, presenta dos retos: el del acercamiento de sus tesoros culturales a su público potencial y el de las mediaciones pedagógicas para la comprensión a su público real.

El advenimiento de los sistemas de comunicación digitales a través de redes de ordenador, y específicamente Internet, están creando un nuevo modelo de presentación y accesibilidad para museos y centros de divulgación científica, redefiniendo su papel como instituciones dedicadas a aumentar el grado de percepción pública de la ciencia y de la tecnología. Surgen, por lo tanto, las cuestiones de cómo utilizar el ambiente virtual como medio educacional, y cuáles son los tipos de aprendizaje más adecuados pra Internet, especialmente en lo que toca a la educación científica. Se trata, entonces, de analizar de forma crítica cuáles son las posibilidades y limitaciones únicas de Internet para los museos científicos (en el apéndice I citamos algunos de los museos de ciencia presentes en Internet, a título de ejemplo).

En el presente artículo, a partir de los conceptos de museo científico y de museo virtual, buscamos describir el estado del arte en cuanto a los contenidos, recursos y características del museo virtual, además de comentar cuestiones de carácter práctico en la operación de los museos virtuales. Además, presentamos los resultados de una investigación experimental con el objetivo de analizar cómo los museos de Ibero América están alcanzando el estadio de "museo virtual", a partir de esta identificación previa de estos contenidos, funcionalidades y acercamientos al museo virtual, con especial énfasis en nuestra realidad. Se trata, en última instancia, de verificar cómo están utilizando Internet para alcanzar nuevos objetivos, sobretodo en el plan pedagógico, y redefinir su misión frente a la sociedad.

En un nivel básico, el museo virtual permitiría el acceso remoto, evitando los gastos de viaje involucrados en una visita al museo real y personas que de ninguna otra manera podrían apreciar el museo ganan una oportunidad de esta manera, además de que se les motive para una posible visita, en cuanto surja la posibilidad (Bowen, 1998). El mismo raciocinio se aplica a algunas audiencias de personas discapacitadas que pueden tener mucha dificultad o incluso incapacidad de visitar las instalaciones físicas de un museo. Ya 
en el ámbito de la investigación académica los catálogos digitalizados y las bases de datos permiten la realización de investigaciones a través de acceso remoto a estos y otros recursos distribuidos globalmente. De todas maneras, solamente si el propio museo -en contraposición a sitios Web creados por entusiastas, instituciones oficiales de turismo o gubernamentales o asociaciones regionales de museos- se encuentra involucrado en la creación de su contrapartida virtual, puede resultar en que la visita virtual resulte en una experiencia más allá del nivel superficial y alcanzar los objetivos de marketing o educativos planteados

Además, los museos virtuales, poseen el potencial de trascender las limitaciones físicas de espacio y tiempo del museo tradicional, trayendo junto a sí, las barreras potenciales de la organización del contexto, de la conservación y seguridad de las colecciones y de un nuevo papel editorial que los museos deben asumir (Hemmings et al., 1997). Igualmente, modifica el concepto de muestra, porque el museo virtual puede incorporar, tanto elementos diseminados en museos diferentes, como simulaciones de funcionamiento para muestras estáticas. Y, pese su aparente falta de realidad, presentan la posibilidad de reunir texto, imágenes e interactividad, además de proporcionar experiencias personales y grupales, aunque estas últimas se realicen de forma distribuida (Semper, 1998). A su vez, Jackson, $(1997,1998)$ cree que las tres principales oportunidades para el visitante virtual se van a dar en tres aspectos físicos de las redes de comunicación digitales: el acceso a datos y a la información, acceso a teleconferencias y sistemas de discusión y el acceso a aplicaciones computacionales de colaboración. Para este autor, estos procesos incluirían al público de especialistas y no especialistas, permitiendo que el desarrollo de instituciones como los museos científicos se realizara por la propia comunidad y sociedad que les rodea.

La base de las actividades los museos científicos reside en la capacidad de exhibiciones de relativa larga duración, complementadas por debates y otras maneras de intercambiar informaciones e ideas -en oposición a los medios tradicionales como televisión y prensa escrita. Así, debido a su enfoque en exhibiciones, los museos científicos poseen una oportunidad natural para la creación de exhibiciones en línea y deberían actuar como puntos nodales en el acceso público a la ciencia y a la tecnología, mezclando exhibiciones electrónicas con actualizaciones en línea inmediatas con la información más actual del momento, mediando la descubierta de información relativas a la actualidad científica, así como de los conceptos por detrás de ellos (Jackson, 1997).

Además, los museos virtuales poseen el potencial de reconceptualizar su relación con grupos, personas y comunidades, utilizando la tecnología disponible en Internet y el espíritu del discurso de la nueva museología, una noción de museología participativa y utilizando el envolvimiento del usuario y los estudios de usuario y el diseño centrado en humanos (Teather, 1998). Por fin, el museo virtual también podría resultar en un museo más democrático, atendiendo a un público cada vez mayor y cada vez más involucrado con el proyecto cultural general, sustituyendo un ambiente controlado, con tarifas de admisión y horarios rígidos por uno de mayor accesibilidad (Schweibenz, 1998).

La discusión académica acerca de los museos virtuales -así como gran parte de la bibliografía utilizada en este trabajo- se ha establecido de manera estable en la serie de conferencias denominadas "Museums and the Web", realizadas a partir de 1997 hasta la actualidad, donde se plantean discusiones acerca de la filosofía, objetivos, gestión y evaluación de sitios Webs de museos más allá de las consideraciones puramente técnicas (Bearman \& Trant, 1999).

\section{Los conceptos de museo y de museo de ciencias}

Huyendo de las definiciones oficiales, Teather (1998) matiza que el concepto de museo se 
encuentra intrínsecamente relacionado con otros tres: el objeto, el significado y la persona. El primer concepto, el de objeto, es quizás el más asociado con el concepto de museo, una vez que se relaciona con su propia evolución histórica. En este sentido, Burcaw (1975) señala que "un museo puede ser definido como una institución sin ánimo de lucro que colecciona, preserva y enseña objetos con fines educativos o estéticos", de forma que la definición estaría estrictamente vinculada al objeto físico". El segundo concepto, el de significado, se encuentra relacionado con la información y con la idea de que los objetos poseen valor por la información intrínseca a ellos, más que por su valor material. Según Macdonald y Alsford (1992) "todos los museos están, en su nivel más fundamental, involucrados con información: su generación, su perpetuación, su organización, su diseminación". Esta concepción implica la transformación del museo de repositorio o receptáculo pasivo a un recurso de información. El tercero de estos conceptos, el relacionado con las personas, deviene del hecho de que éstas son el centro mismo de la experiencia museística, los receptores de los significados que se están transmitiendo en la experiencia de visita. De esta manera, se podría decir que son las personas que dan sentido a la existencia del museo y que se aprovechan de sus actividades de investigación, inspiración, educación o entretenimiento. Esta línea de pensamiento toma principal importancia hoy, con la introducción de la cuestión de los museos virtuales, pues está profundamente relacionada con la concepción de éstos últimos.

A su vez, los museos de ciencia se pueden clasificar tipológicamente con base en sus objetivos, más que en el área temática que representan. Así, los museos que tuvieron sus orígenes en colecciones de curiosidades, máquinas o instrumentos, tenían como objetivo mostrar la ciencia, a través de la enumeración de las realizaciones técnicas de un país en una determinada época. Los museos creados en el primer tercio del siglo XX tenían como objetivo demostrar la ciencia, "añadiendo a la conservación y presentación del patrimonio científico y técnico una decidida voluntad pedagógica y cultural". Por fin, a partir de los sesenta una nueva tendencia ha orientado cada vez más un gran número de instituciones, con el objetivo de comunicar la ciencia, "centros de percepción" en los cuales se despertaría la curiosidad dotados de atractivo estético y pedagógico, también conocidos como centros de ciencia (Pérez et al., 1998). Según la definición propuesta por Wagensberg (2000):

Un museo de ciencia (MC) es un espacio dedicado a crear, en el visitante, estímulos a favor del conocimiento y del método científico (lo que se consigue con sus exposiciones) y a promover la opinión científica en el ciudadano (lo que se consigue con la credibilidad y prestigio que sus exposiciones dan al resto de las actividades que se realizan en el museo: conferencias, seminarios y congresos...).

Por otro lado, el papel de un museo científico en una sociedad democrática se sitúa como interfaz y mediador de cuatro sectores específicos relacionados con el sistema de ciencia y tecnología de un país: la sociedad misma entendida como el ciudadano de a pie que se beneficia y sufre la ciencia, la comunidad científica donde se crea el conocimiento científico, el sector productivo y de servicios donde se usa la ciencia y la administración donde se gestiona la ciencia. En esta red de relaciones, uno de los aspectos más discutidos del papel de los museos científicos se encuentra relacionado con cambiar la relación del público general con la ciencia, caracterizada por una falta de interés y hostilidad en relación con a la ciencia y por un bajo nivel de comprensión de la ciencia y sus métodos.

\section{El concepto de museo virtual}

El concepto básico por detrás del museo virtual sería, según MacDonald (1992), el de "connectedness"[1] o "el recurso básico del museo virtual, con la presentación interrelacionada e interdisciplinaria de la información museística, con el auxilio del 
multimedia, capacidad de trascender el museo físico en la habilidad de presentar información". El concepto del museo virtual demuestra cómo las limitaciones impuestas por el método tradicional de organizar y presentar información puede ser superado en el contexto de las visitas al museo. Rápidamente, el Museo Virtual proporciona múltiples niveles, perspectivas y dimensiones de información acerca de determinado tópico: proporciona no solo multimedia (texto, imágenes visuales a través de fotografías, ilustraciones o video, y audio), sino también información que no ha sido filtrada por estos métodos tradicionales.

De forma que el gran diferencial del museo virtual residiría en la capacidad de establecer vinculaciones entre los objetos, dar la oportunidad al visitante de centrarse en sus tópicos de interés y establecer un diálogo interactivo con el museo, lo que implicaría el cambio de paradigma desde el enfoque en la colección -en el objeto- hacia la audiencia -las personas- conforme comentado anteriormente. Así, además de la capacidad de realizar interconexiones entre los bloques de información, uno de los principales requisitos de los museos virtuales sería el reconocimiento de que el ambiente virtual es interactivo y que por lo tanto, el enfoque se encuentra en el usuario (Bearman, 1995). Sintetizando todas estas matizaciones, Schweibenz, (1998) propone la siguiente definición:

El museo virtual es una colección de objetos digitales lógicamente relacionados compuestos de una variedad de medios, y, debido a su capacidad de proporcionar "connectedness" y varios puntos de acceso, se presta a trascender los métodos tradicionales de comunicación y la interacción con el usuario es flexible en relación con a sus necesidades e intereses; no posee lugar o espacio real, sus objetos y la información relacionada pueden diseminarse a través de todo el mundo.

\section{Características y tipología de los museos virtuales}

Una clasificación de la tipología de los museos virtuales frecuentemente utilizada es la realizada por Piacente (1996), que identifica tres tipos de páginas Web que representan a los museos en Internet. La primera categoría sería el "folleto electrónico", en su esencia un formato de propaganda como los folletos utilizados en la promoción del museo. El folleto electrónico presentaría información básica sobre el museo, como su historia, fotos del exterior y del interior, fotos de algunos contenidos disponibles, horarios de apertura, precios y tarifas y datos de contacto. La segunda categoría sería el "museo en el mundo virtual", o sea, proyección del museo físico en el ambiente virtual, con la representación de planos, información sobre colecciones y exhibiciones, además de exhibiciones en línea. Algunas veces estos tipos de museos utilizan el espacio virtual para archivar exhibiciones retiradas o para enseñar elementos de sus colecciones que no se encuentran disponibles para el público del museo real a través de bases de datos interactivas. Por fin, en los "verdaderamente interactivos", existe alguna relación con el museo físico, pero también se reinventa o se añaden elementos al museo, involucrando de paso a los visitantes en actividades interactivas.

En otro nivel, los museos deberían incentivar la participación y la toma de decisiones por parte del público, potenciando la participación intelectual, la interacción social y permitiendo la síntesis de las informaciones. Un ejemplo, aplicado a los museos y centros de ciencia, serían las encuestas interactivas de evaluación sobre la actitud ante determinados temas científicos, con la oportunidad del visitante de comparar sus respuestas personales con las de población general, aumentando la conciencia de cómo sus propias actitudes se encajan dentro de un contexto más amplio.

Según Roberts (1997), estas diferentes perspectivas, y el establecimiento de tensiones y conflictos entre distintas concepciones permitirían la reconstrucción de visiones del mundo, una vez que la diferencia entre la cultura del visitante y la cultura presentada en 
el museo pasaría por un proceso de negociación y de construcción del significado. Esto supondría un cambio del paradigma "lógico-científico" (experto) a un paradigma "narrativo", en el cual las múltiples interpretaciones del visitantes sustituyen la "verdad" incuestionable.

\section{Las relaciones entre el museo físico y el museo virtual}

De todas las discusiones que cercan el concepto del museo virtual, una de las que más cuestionamientos suscita es si es posible tener una experiencia significativa o "real" visitando el museo virtual. Aunque los medios digitales y las redes de comunicación puedan ampliar y dar nuevos significados a la información que un museo proporciona a su público, la cuestión de la suplantación todavía es muy fuerte como para olvidarse. El museo virtual debería proporcionar experiencias multimedia auténticas en su dominio, pero sin aspirar a la autenticidad del objeto real, que por propia definición no puede ser mediada. Nunca habrá un "museo virtual" en el sentido completo de la palabra, una vez que la visita virtual es fundamentalmente una experiencia mediática, y no una experiencia museística[2]:

"el elemento museológico y museográfico prioritario es la realidad, esto es, el objeto real o fenómeno real. El texto, la voz, la imagen, el juego, la simulación, la escenografía o los modelos de ordenador son elementos prioritarios en otros medios, como las publicaciones, la TV, el cine, el parque temático, las clases, las conferencias, el teatro, etc., pero en museografía son sólo elementos complementarios. Una exposición nunca debe basarse en tales accesorios, es decir, una exposición de accesorios de la realidad puede ser muchas cosas, pero no una exposición" (Wagensberg, 2000)

En tal caso, dado la imposibilidad del museo virtual de sustituir el museo real, al museo virtual le quedarían dos opciones: simplemente replicar los contenidos existentes, sin llegar nunca a alcanzar el mismo nivel de experiencia, o por otro lado, extender el museo a "algo más"

En ambos los casos, la representación del museo real en la Web, respondería a los distintos grado de virtualización posibles. El grado máximo de virtualización se encontraría en el momento en que el museo virtual facilitase una experiencia única de visitante que no se puede replicar en el museo físico.

\section{Contenidos del museo virtual}

\subsection{Folleto virtual}

Los primeros museos en adentrarse en el mundo virtual se han basado en un medio preexistente, el folleto de divulgación. El folleto posee además, una forma bastante adaptable para representarse en la Web en la constitución de lo que se podría llamar "la máquina de propaganda personal", dado algunas características que ambos comparten: la distribución de un documento a una audiencia lo más amplia posible, la existencia de información visual y textual y la facilidad de publicación (Streten, 2000). Además, como elemento de primera impresión, el museo virtual debería reflejar de forma precisa la institución que representa y actuar como un mensaje de bienvenida a las audiencias tradicionales y en línea (Hazan, 1997), otro de los elementos característico de este tipo de documento de divulgación.

\subsection{Colecciones, exhibiciones y experimentos en línea}

Aún con la existencia de ciertas limitaciones, el concepto de permanencia ý preservación de los objetos nos lleva directamente al de archivo o colección digital, donde la 
digitalización no se limita a imágenes, sino se extiende a texto, información tridimensional, sonidos y video. Otro concepto de la colección en línea frecuentemente destacado en la literatura se refiere a que mientras existen exhibiciones permanentes en muchos museos de ciencia, la limitación de espacio y la política del museo limitan el tiempo que determinadas exhibiciones puedan estar a disposición del público. En el ámbito virtual, sin embargo, tales limitaciones prácticamente no existen (Orfinger, 1998). Las colecciones en línea suelen disponer de mecanismos de búsqueda vinculados a bases de datos y permitir el flujo de navegación desde la colección general, pasando por una versión reducida del objeto hasta una imagen ampliada del objeto acompañado de una leyenda o explicación. Esta metodología mimetiza el método de presentación utilizado por los museos físicos tradicionales: la información centrada en el objeto y segregada en galerías o áreas temáticas, limitaciones innecesarias en el ambiente virtual (Donovan, 1997).

A su vez, una exhibición en línea debería ser algo más que la representación en formato digital de las colecciones del museo; una exhibición eficaz en el museo real debe revelar las relaciones subyacentes entre distintos objetos y su contexto y debe ser programada para crear una experiencia cultural relevante. Entretanto, se pueden observar dos diferencias fundamentales entre una exhibición en línea y una exhibición física. La primera, se deriva del hecho de que un ordenador nunca será capaz de replicar o simular la experiencia sensorial que el contacto con el objeto puede suponer. Luego, que la visita a un museo virtual suele ser una experiencia más solitaria que la visita a un museo real, que suele ser una experiencia social. Debido a estas diferencias, más que una representación digital del espacio físico, los museos virtuales deberían sacar partido de algunas calidades únicas de los medios digitales para crear experiencias atractivas y novedosas.

\subsection{Visitas virtuales y ambientes de realidad virtual}

Según Barbieri y Paolini (2000), las visitas virtuales se podrían clasificar según tres tipos. El primero, más sencillo y asequible, estaría constituido por un conjunto de páginas Web dispuestas de forma lógica y con el objetivo de proveer una visita guiada a las instalaciones del museo. El segundo tipo utilizaría ambientes en 3D navegables, por ejemplo utilizando la tecnología Quicktime VR y que proporcionan una sensación mínima de presencia pero que resultan en una experiencia solitaria para el visitante. Por último, una visita virtual en un ambiente de simulación 3D ofrecería una ampliación del real permitiendo al visitante un mayor grado de libertad y que este interactuara con modelos y objetos, "tocando libremente", y con la posibilidad de interacción con otros visitantes virtuales. El grado máximo de visita virtual vendría en cuanto este tipo de ambiente permitiera la interacción en tiempo real entre los visitantes virtuales.

\subsection{Materiales y actividades educativos}

La visión más común de la utilización de Internet para la creación de materiales educativos se refiere a la generación de actividades para que los profesores desarrollen contenidos curriculares con sus alumnos, de forma que "el alumno reconstruya la red de saberes interdisciplinarios que estructuran el fenómeno. Cada una de estas actividades puede extenderse y complementarse en las lecciones de matemáticas, ciencias, en el uso de las TIC e Internet, y en otros conocimientos generales". Según Honeyman (1996), las experiencias educativas en línea podrían ser mucho más significativas desde el punto de vista educativo una vez que las actividades en línea de los museos y centros virtuales se están diseñando para que los visitantes exploren e interactúen con los fenómenos utilizando los objetos de sus colecciones, la mayoría de ellos están incorporando distintas estrategias para responder a la diversidad de conocimientos y experiencias de los visitantes. Sus exhibiciones virtuales son abiertas y flexibles y los recursos en línea 
buscan favorecer el espíritu investigativo con actividades y proyectos para desarrollar en forma individual o en grupo. Así, las nuevas tecnologías aplicadas a las actividades pedagógicas suponen un mejor aprovechamiento del tiempo disponible, el aprendizaje individualizado y el seguimiento del progreso de forma individualizada, las posibilidades de aplicar estrategias de aprendizaje colaborativo, el reconocimiento de la diversidad cultural y la creación de una conciencia de responsabilidad social.

En la cuestión de cómo facilitar la alfabetización científica el museo debería ofrecer recursos didácticos atractivos -que se puedan utilizar antes y después de la visita- y posibilitar la reflexión y la comparación, vinculando de esta forma el grupo de alumnos con la sociedad. Recursos como juegos - crucigramas, asociaciones entre palabras, rompecabezas y sopas de letras - clasificados según niveles de dificultad constituirían algunos de los elementos disponibles para los alumnos (Correa \& Ibáñez, s/d).

Por otro lado, la enseñanza en ciencias debería "proporcionar oportunidades para explorar el mundo, aplicar principios científicos, evaluar y analizar datos y hacer conexiones entre estas exploraciones, sus vidas personales, sus comunidades y otras partes del currículo". Vinculados a estos objetivos se encuentran el reconocimiento de la importancia de la ciencia, la resolución de problemas de la vida cotidiana a través del método científico, el desarrollo del pensamiento crítico y la consideración de las conexiones entre ciencia, tecnología, historia y sociedad (Dede \& Ruess, 2000). El asumir el papel de un científico implica el desarrollo y prueba de hipótesis, con la articulación de defensas de su diseño de investigación, la consideración de distintas hipótesis simultáneamente, la prueba de las evidencias, el descarte de hipótesis alternativas y la argumentación persuasiva, con un grado adecuado de confianza (Berger et al. 1994). Así, el aprendizaje activo - con el alumno interactuando con una gran variedad de recursos, con la mezcla de experimentación y de tutoría por expertos, procesos éstos complementados a través de la interacción social con otros aprendices en el proceso de construcción del conocimiento busca sobretodo la práctica científica en un contexto social (Edelson, Pea, and Gomez 1996). Al mismo tiempo, se reconocen algunas demandas del proceso de enseñanzaaprendizaje, con la adopción de una pedagogía constructivista, enfoque en el aprendizaje en grupo y del papel del profesor como guía, además del papel del multimedia en favorecer distintos estilos de aprendizaje y del aprendizaje orientada a proyectos. (Pea, Edelson, and Gomez 1994)

Teniendo en cuenta estas consideraciones, destacan los experimentos en línea y las simulaciones también tendrían un importante papel en el museo virtual, bajo la justificación de que "muchos sofisticados procesos científicos, técnicos e industriales sólo es posible simularlos mediante las ricas convergencias multimedia interactivas, que, además, permiten reconstruir la evolución histórica" (Moreno, 1997). Freeth (1998) menciona que la posibilidad de que los experimentos virtuales hagan uso de simuladores, por ejemplo, en exhibiciones que ilustren la física newtoniana[3]. Asimismo, se podría aplicar a la disección de artefactos físicos, a la creación de soluciones de diseño colaborativos y a la manipulación de artefactos físicos (Friedlander, 1998). También cabe hacer una distinción entre lo que son las demostraciones, que tienen como objetivo la ilustración de conceptos, los elementos de evaluación, como pueden ser los tests de múltiple elección, aleatorios o adaptativos y la experimentación propiamente dicha, con la manipulación de parámetros y la visualización interactiva por parte del usuario. Para Carnevale (2003), la cuestión supone un cambio de paradigma, desde la transferencia de información en contraposición al espíritu investigativo en los laboratorios virtuales. Así, las simulaciones, debido a sus características de la utilización de multimedia, de respuesta instantánea al usuario, de interactividad y de animación ofrecen beneficios cognitivos a los aprendices, debido al envolvimiento de más sentidos en el proceso de aprendizaje, a la reducción de la carga de manipulación mental, tareas cognitivas relacionadas con 
procesos o con visualizaciones de estructuras en tres dimensiones y a la reducción del ruido, según las varias teorías propuestas para el aprendizaje multimedia (Gaddis, 1999)

Por último, a través de las actividades pedagógicas, el museo debería establecer relaciones a largo plazo con su audiencia y con la comunidad a que sirve. El ejemplo más contundente en este sentido es el proyecto STEM, desarrollado por el Science Museum, que anima a los estudiantes y sus profesores a crear sus propias perspectivas, a través de proyectos, y recursos educativos relacionados con el museo, y hacerlos disponibles a través de Internet. Entretanto, la publicación de los recursos creados se realiza en sus propios servidores, aunque sean registrados y catalogados en una base de datos del museo. Los recursos enviados demandan una evaluación, además de tareas de gestión y mantenimiento en la base de datos, sobretodo para la detección de información que caduca o de sitios Web que son trasladados de sitio (Jackson et al. 2002)

\section{Análisis de los contenidos informativos y educativos de los museos de ciencia en Internet en Ibero América}

Con base en el panorama teórico y práctico descrito se ha realizado una investigación cuyo objetivo ha sido analizar cómo los museos y centros de ciencia de Ibero América, están utilizando las nuevas tecnologías de información y comunicación, particularmente Internet y la World Wide Web para constituir el llamado "museo virtual" y cumplir su misión de informar, educar y aumentar la percepción pública sobre la ciencia y la tecnología. Pretendemos, de esta forma, realizar un análisis sistemático y profundo, identificando cuáles son los contenidos, funcionalidades y abordajes del museo virtual, con especial énfasis en nuestra realidad.

Nuestra muestra ha consistido de 63 museos virtuales, de países de Iberoamérica, América del Norte y Europa Occidental. Para cada categoría superior de análisis (Información general sobre el museo virtual", "Información sobre el museo físico", "El museo virtual") se ha calculado un índice general, a través del porcentaje de las ocurrencias sobre el total de categorías analizadas, para cada caso. Un índice calculado hasta $25 \%$ se ha considerado como bajo, entre 25 y $75 \%$ se ha considerado mediano y mayor que $75 \%$ se ha considerado alto. Los datos se han agrupado según zonas geográficas de análisis, para su interpretación. Además de los índices generales, también se ha aplicado la misma clasificación para cada variable analizada individualmente.

Para la obtención de la información se ha utilizado un análisis "heurístico", definido como la inspección sistemática de determinados elementos -en el caso, nuestras variables, en función de una serie de categorías establecidas previamente realizado para cada museo visitado- y de la verificación de su ocurrencia o no. Se ha utilizado un instrumento de investigación, con la definición de una serie de categorías que describen los contenidos, servicios y funcionalidades presentes en los museos virtuales, además de las tecnologías informáticas utilizadas. El instrumento está compuesto de 103 categorías, agrupadas en cinco grandes bloques de más alto nivel: además de las categorías ya mencionadas se han utilizado "Elementos de Identificación" y "Tecnologías utilizadas". El instrumento se ha construido con base en instrumentos realizados en otras investigaciones, incorporando además algunos elementos adicionales identificados en el estado del arte de los museos virtuales.

A través de este análisis hemos podido constatar, empíricamente, que en la actualidad existe una gran diferencia entre los museos virtuales de Ibero América, sea en Latinoamérica o en la Península Ibérica, en comparación aquellos encontrados en países como Estados Unidos, Reino Unido e Italia. Es más, los museos virtuales de Ibero América no solo no aprovechan las potencialidades de los museos "verdaderamente" interactivos, sino que tampoco trasladan la información del museo físico a Internet de una 
manera adecuada, como se ha comprobado a través del análisis de los índices generales y de las categorías de análisis por separado. también se nota una ausencia general de las iniciativas relacionadas con la enseñanza de las ciencias y de la asociación con el sector educacional. De esta manera, los museos y centros de ciencia de Ibero América no están trasladando -y tampoco ampliando- su misión educativa a Internet, y a las posibilidades que la comunicación digital, las estrategias de trabajo colaborativo y la enseñanza a distancia ofrecen, fruto quizás de una excesiva preocupación con la información sobre el museo físico y con la difusión de las informaciones que se transmiten tradicionalmente a través de otros medios.

\section{Conclusión}

En su conjunto, el tradicionalismo y la ausencia de experiencias realmente innovadoras nos puede llevar a creer que los museos de ciencia en Iberoamérica están perdiendo una oportunidad única, de experimentar, innovar y quizás, redefinir su papel y su misión frente a la sociedad. Se demandan, por lo tanto, iniciativas de colaboración para la propulsión del concepto de museo virtual, por ejemplo a través de la creación de un metamuseo o meta-centro de información, que funcionara como un punto de acceso común a la información de los distintos museos y que además establecería la base institucional para la realización proyectos comunes, para la obtención de fondos y para la concienciación en relación con las posibilidades educativas del museo virtual.

Por otro lado, el concepto de museo virtual abre innumerables posibilidades de acceso a manifestaciones y creaciones culturales que ni la escuela ni los textos pueden resolver por sus limitaciones como instrumentos de acceso a la cultura, al tiempo que complementa de manera relevante las posibilidades de formación que tanto las aulas como los textos pueden promover. En este punto también destacamos la utilización de tecnologías informáticas aplicadas al ámbito de la educación de las ciencias, como por ejemplo las demostraciones interactivas multimedia y los experimentos virtuales que, utilizados en conjunto con herramientas de colaboración y comunicación, pueden acercar la actividad didáctica a la práctica científica, acercándose a la reforma educativa demandada en la actualidad.

\section{Referencias}

BARBIERI, T., Paolini, P. (2000). Cooperative visits for the museum WWW sites a year later: evaluating the effect. Museums and the Web 2000. Selected papers. Pittsburgh: Archives and Museum Informatics.

BEARMAN, D. (1995). Museum Strategice for Success on the Internet. Museum collections and the Information Superhighway Conference.

BEARMAN, D., TRANT, J. (1999). Museum Internacional, 51, n. 4: 20-3.

BERGER, C F.; LU, C.R.; BELZER, S.; VOSS, B.E. (1994). Research on the uses of technology in science and education, en GABEL, Dorothy L.(ed.) Handbook of research on science teaching and learning : a project of the National Science Teachers Association, New York: Mcmillan.

BOWEN, J. (1998). Virtual visits to virtual museums. Museums and the Web 98. Conference proceedings. Pittsburgh: Archives and Museum Informatics.

BURCAW, G. E. (1975). Introduction to museum work. Nashville: American Association for State and Local History. 
CARNEVALE, D. The virtual lab experiment. The Chronicle of Higher Education, 49(21), 2003, A30.

CORREA, J., IBÁÑEZ, A. (s/d). Internet, enseñanza y difusión de la ciencia. Quaderns Digitals, 24.

DEDE, C., RUESS, K. (2000). Museum-Related Multimedia and Virtual Environments for Teaching and Learning Science. CILT 2000: Technology, Equity, and K-14 Learning.

DONOVAN, K. (1997). The best of the intentions: public access, the Web \& the evolution of museum automation. Museums and the Web 97. Selected papers. Pittsburgh: Archive and Museum Informatics.

EDELSON, D. C., ROY D. P., GOMEZ, L. (1996). Constructivism in the collaboratory, en Wilson B.G. (ed.) Constructivist learning environments: Case studies in instructional design, Englewood Cliffs: Educational Technology Publications, p, 151-154.

FERNSTRÖM, M., BANNON, L. (1997). Enabling technology for museum visitors: issues and experiences. Museums and the Web 99. Selected papers. Pittsburgh: Archives and Museums Informatics.

FREETH, M. (1998). Hands online. Museums and the Web 98. Conference proceedings. Pittsburgh: Archives and Museum Informatics.

FRIEDLANDER, L. (1998). Models for a new visitor-centered museum: using the web to create community and continuity for the museum visitor. Museums and the Web 98. Conference procedings. Pittsburgh: Archives and Museum Informatics.

GADDIS, B. (2000). Learning in a virtual lab: Distance education and computer simulations. Manuscrito no publicado, University of Colorado.

HAZAN, S. (1997). The virtual museum. The Israel Museum Journal, XV, Summer.

HEMMINGS, T. (1997). Situated knowledge and the virtual science and industry museum. Museums and the Web 99. Selected Papers. Pittsburgh: Archives and Museum Informatics.

HONEYMAN, B. (1996). Science centres and the World Wide Web. Proceedings of the 3rd International Interactive Multimedia Symposium. Canning Bridge: Promaco Conventions .

JACKSON, R. (1997). The virtual visit: towards a new concept for the electronic science centre. Here and now. Contemporary science and technology in museum and science centres. Londres: Science Museum.

JACKSON, R. (1998). Using the Web to change the relation between a museum and its users. Museums and the Web 98. Conference procedings. Pittsburgh: Archives and Museum Informatics.

JACKSON, R., BAZLEY, M.; PATTEN, D.; KING, M. (2002). Using the Web to change the relation between a museum and its users. Museums and the Web 2002. Selected papers. Pittsburgh: Archives and Museum Informatics.

KYDD, S., MACKENZIE, D. (1997). Going on-line: moving multimedia exhibits onto the Web. Museums and the Web 97. Selected papers. Pittsburgh: Archives and Museum Informatics. 
MACDONALD, G. (1992) Change and Challenge: Museums in the Information Society, en KARP, I. (ed.) Museums and Communications - The Politics of Public Culture, ed. I Karp. Washington, D.C.: Smithsonian Institution Press.

MACDONALD, S., SILVERSTONE R. (1992). Science on display: the representation of scientific controversy in museum exhibitions. Public Understanding of Science, 1 (1), 6988 .

MINTZ, A. (1998). Media and museums: a museum perspective. The virtual and the real: the media and the museum, ed. Selma Thomas and Mintz Ann, 19-34. Washington, D.C.: American Association of Museums.

MORENO, I. (1997). Multimedia y museos en España. De prohibido tocar a toque por favor. Cinevideo, 20.

ORFINGER, B. (1998). Virtual science museums as learning environments: interactions for education. Informal Learning Review, 33, 8-13.

PEA, R. D., EDELSON, D., GOMEZ, L. (1994). The CoVis Collaboratory; high school sicence learning supported by a broadband educational network with scientific visualization, videoconferencing and collaborative computing. Annual Meeting of the American Educational Research Association.

PÉREZ, C., DÍAZ, M. P., ECHEVARRÍA, I., MORENTIN, M., CUESTA, M. (1998). Centros de ciencia : espacios interactivos para el aprendizaje. Bilbao: Universidad del País Vasco, Servicio Editorial.

PIACENTE, M. (1996). Surf's up: Museums and the world wide web. University of Toronto.

ROBERTS, L. (1997). From Knowledge to Narrative: Educators and the Changing Museum. Washington, D.C.: Smithsonian Institution Press.

SCHWEIBENZ, W. (1998). The "Virtual Museum": New Perspectives For Museums to Present Objects and Information Using the Internet as a Knowledge Base and Communication System. Knowledge Management und Kommunikationssysteme. Workflow Management, Multimedia, Knowledge Transfer. Proceedings des Internationalen Symposiums für Informationswissenschaft (ISI '98) .

SEMPER, R. (1998). Bringing authentic museum experience to the web. Museums and the web 1998. Conference Proceedings. Pittsburgh: Archives and Museum Informatics.

STRETEN, K. (2000). Honoured guests: towards a visitor centered Web experience. Museums and the Web 2000. Selected papers. Pittsburgh: Archives and Museum Informatics.

TEATHER, L. (1998). A museum is a museum...or is it? Exploring museology and the $w e b$. Museums and the Web 98. Conference proceedings. Pittsburgh: Archives and Museum Informatics.

TINKLER, M. (1998). Online Exhibitions: A Philosophy of Design and Technological Implementation. Museums and the Web 98. Conference proceedings. Pittsburgh: Archives and Museum Informatics.

WAGENSBERG, J. (2000). Principios fundamentales de la museología científica moderna. Alambique: Didáctica de las Ciencias Experimentales, VII (26). 
WHALLEY, P. 1995. Imagining with multimedia. British Journal of Educational Technology 26 (3), 194-204.

Apéndice I - Algunos museos y centros de ciencia en Internet

\section{Alemania}

Deustche Museum

http://www.deutsches-museum.de

\section{Australia}

Questacon - The National Science \& Technology Centre

Canberra

http://www.questacon.edu.au

\section{Brasil}

Estaçao Ciência - Universidade de Sao Paulo

Sao Paulo

http://www.eciencia.usp.br

\section{Canadá}

Ontario Science Centre

http://www.ontariosciencecentre.ca

\section{Chile}

Museo Interactivo Mirador

http://www.mim.cl/

\section{Colombia}

Maloka

Bogotá

http://www.maloka.org

\section{Dinamarca}

Experimentarium

http://www.experimentarium.dk

\section{España}

Museos Científicos Coruñeses

http://www.casaciencias.org

Parque de las Ciencias

http://www.parqueciencias.com

Museo Miramon - Kutxa Espacio de la Ciencia http://www.miramon.org

Museo Nacional de Ciencias Naturales (CSIC) 
http://www.mncn.csic.es

\section{Estados Unidos}

Exploratorium - The museum of art, perception and human perception http://www.exploratorium.com

The Franklin Institute http://sln.fi.edu/

Museum of Science http://www.mos.org

Science Museum of Minnesota http://www.smm.org

Oregon Science and Industry Museum http://www.omsi.edu

National Museum of Natural History http://www.mnh.si.edu

National Air and Space Museum http://www.nasm.si.edu

\section{Finlandia}

Heureka

http://www.heureka.fi

\section{Francia}

Cité des Sciences et de l'Industrie (La Villete)

http://www.cite-sciences.fr

\section{Italia}

Museo Nazionale de la Ciencia y de la Tecnica Leonardo da Vinci http://www.museoscienza.org

Science Centre - Immaginario Scientifico http://www.immaginarioscientifico.it/

Instituto e Museo di Storia della Scienza http://www.imss.firenze.it/

\section{Holanda}

New Metropolis Science Centre Association http://www.e-nemo.nl/

\section{México}

Centro de Ciencias Explora

http://www.explora.edu.mx/ 


\section{Portugal}

Pavilhão do Conhecimento - Ciência Viva

http://www.pavconhecimento.mct.pt/

\section{Reino Unido}

The National Museum of Science and Industry - Science Museum

http://www.sciencemuseum.org.uk/

Techniquest

http://www.tquest.org.uk

Glasgow Science Center

http://www.gsc.org.uk

\section{Notas}

[1] No existe traducción para este término, pero se referiría a la capacidad de establecer conexiones -entre la propia información y entre los usuarios.

[2] Una excepción interesante serían los museos dedicados a la computación per se, los museos científicos y los museos de niños, que frecuentemente exploran la computación como tema. En este contexto especial "el virtual casi literalmente es el real" (Mintz 1998).

[3] También es interesante destacar el hecho de de que la experimentación virtual permite una experiencia que trascenda lo real. En este sentido el "Microscopio Virtual", desarrollado por la Open University de Reino Unido consiste de un aparato virtual que permite a los estudiantes voltear una muestra de roca, mientras la visualizan en luz polarizada plana y transversal al mismo tiempo, hecho que lo que no es posible en un microscopio convencional (Whalley, 1995).

(C) Ediciones Universidad de Salamanca 